\title{
Experimental Tests of an Infrared Video Bolometer on Alcator C-Mod
}

M.L. Reinke, ${ }^{1}$ J. Terry, ${ }^{2}$ G.G. van Eden, ${ }^{3}$ B.J. Peterson, ${ }^{4,5}$ K. Mukai, ${ }^{4,5}$ T.K. Gray, ${ }^{1}$ and B. Stratton ${ }^{6}$

1) Oak Ridge National Laboratory, Oak Ridge, TN, USA

${ }^{2)}$ MIT Plasma Science and Fusion Center, Cambridge, MA, USA

${ }^{3)}$ Dutch Institute for Fusion Energy, Eindhoven, The Netherlands

4) National Institute for Fusion Science, Toki, Japan

5) SOKENDAI (The Graduate University for Advanced Studies), Toki, Japan

${ }^{6)}$ Princeton Plasma Physics Laboratory, Princeton, NJ, USA

(Dated: 14 September 2018)

A prototype of an infrared imaging bolometer (IRVB) was successfully tested on the Alcator C-Mod tokamak at the end of its 2016 campaign. The IRVB method interprets the power radiated from the plasma by measuring the temperature rise of a thin, $\sim 2 \mu \mathrm{m}$, Pt absorber that is placed in the torus vacuum and exposed, using a pinhole camera, to the full-spectrum of plasma's photon emission. The IRVB installed on C-Mod viewed the poloidal cross-section of the core plasma and observed Ohmic and ICRF-heated plasmas. Analysis of total radiated power and on-axis emissivity from IRVB is summarized, and quantitative comparisons made to data from both resistive bolometers and AXUV diodes. IRVB results are clearly within a factor of two, but additional effort is needed for it to be used to fully support power exhaust research. The IRVB is shown to be immune to electromagnetic interference from ICRF, which strongly impacts C-Mod's resistive bolometers. Results of the bench-top calibration are summarized, including a novel temperature calibration method useful for IRVBs.

\section{INTRODUCTION}

To scale present successes in magnetic confinement of high temperature plasmas to devices that would be attractive for commercial generation of power is known to require integrated solutions for power exhaust and particle control $(\mathrm{PEPC})^{12}$. The underlying physics can be studied in present devices and requires dedicated diagnostics, both to demonstrate feedback control and to provide sufficient data to validate theory and modeling. One paradigm for PEPC solutions requires seeding the plasma with extrinsic impurities which remain partially ionized and exhaust heat from the plasma isotropically through short wavelength photon emission. This allows plasma facing components (PFC) to avoid overheating and lowers boundary temperatures to facilitate a range of processes which, working together, can reduce PFC erosion.

Presently, there are uncertainties in the required amount and spatial distribution of extrinsic impurities when comparing results of core and boundary modeling with experimental multi-chord resistive bolometry ${ }^{34}$. The infrared video bolometer (IRVB) concept for measuring plasma radiation may offer a means to improve the spatial coverage of divertor radiation measurement. IRVB systems have been demonstrated on multiple tokamak $^{567}$ and helical ${ }^{8910}$ devices, but have seen limited cross-comparison with other methods of radiation measurement. This work outlines the testing of a prototype IRVB system on Alcator C-Mod ${ }^{11}$. It details new methods of bench-top calibration in Section III and shows comparisons to existing resistive bolometer (RB) and AXUV diode measurements in Section IV. A brief overview of the IRVB method and the specific hardware utilized are first discussed in Section II. This work demonstrates that the IRVB method can resolve time- evolving core radiated power density profiles (e.g. units of $\mathrm{MW} / \mathrm{m}^{3}$ and total core radiated power (e.g. units of MW) in a tokamak to within a factor of two of proven systems, and argues the accuracy needed to fully support PEPC research, $<10 \%$, may be feasible with improved calibration. A clear advantage of IRVB over resistive bolometry is the former's avoidance of electromagnetic interference, shown in this case by the lack of noise from the $78-80 \mathrm{MHz}$ ion cyclotron range of frequency (ICRF) heating used on C-Mod.

\section{IRVB AND DIAGNOSTIC OVERVIEW}

Bolometers used on magnetic confinement devices work by measuring the temperature rise of an absorber exposed to wide-spectrum radiation from the plasma using a simple pinhole camera. Resistive bolometers, common to many tokamaks ${ }^{1213}$, are electrical transducers of this temperature rise, while the IRVB is an optical transducer, inferring the temperature rise of the absorber from its infrared (IR) emission (typically in the $1-10 \mu \mathrm{m}$ range) using research-grade IR cameras. RB sensors are discrete elements while IRVB is typically done using a larger, often $2 \mathrm{D}$, continuous absorber where spatial diffusion leads to cross-talk. The local power density deposited on the absorber by the plasma is derived from the absorber's temperature measurement using a conductive and radiative power balance ${ }^{14}$,

$$
P=k t_{f}\left[\frac{1}{\kappa} \frac{\partial T}{\partial t}-\left(\frac{\partial^{2} T}{\partial x^{2}}+\frac{\partial^{2} T}{\partial y^{2}}\right)\right]+2 \varepsilon \sigma_{S B}\left(T^{4}-T_{0}^{4}\right)
$$

where $k t_{f}$ is the product of the absorber conductivity, $k$, and thickness, $t_{f} . \kappa$ is the thermal diffusivity, $\varepsilon$ is the emissivity and $\sigma_{S B}$ is Stefan-Boltzmann constant. $P$ is 
the power density deposited on the foil (e.g. $\left.\mathrm{W} / \mathrm{m}^{2}\right)$. The $T_{0}$ is the environmental temperature, which for this analysis is taken to be the pre-shot foil temperature, which assumes system is in thermal equilibrium and does not change during the $<2$ second C-Mod discharge. The $(x, y)$ dependence of $k t_{f}, \kappa$, and $\varepsilon$ are determined using a known, localized heating source, $P(x, y)$, and adjusting those three parameters until a match is reached with measured temperature, using (1).

Many details of this IRVB construction can be found in $^{15}$, as it was originally designed and installed on NSTXU. However IR camera DAQ problems prevented its use prior to the early termination of the NSTX-U campaign in July 2016. The system was designed to give a collimated, poloidal view due to a requirement to view through a gap in the NSTX-U passive plates. Its installation on Alcator C-Mod exploited an existing IR camera (a Cedip Titanium 550M) that was being used to measure heat fluxes on the inner divertor target. It was viewing through a sapphire view-port mounted behind a gate valve. The vacuum build shown in Figure $1 \mathrm{a}$ in ${ }^{15}$ was installed in place of the sapphire window, giving the system a 1-D, poloidal view of the C-Mod plasma, viewing radially into the torus. The $7.5 \times 2.7 \mathrm{~mm}$ aperture was located at $(R, Z)=(3.3,0.24) \mathrm{m}$, with the $9.0 \times 7.0 \mathrm{~cm}$ absorber located $19.1 \mathrm{~cm}$ behind the aperture. The longter dimension of the aperture is nominally in the toroidal direction, while the shorter is in the vertical. A full view of the absorber was not possible, due to limited alignment flexibility of the IR camera, viewing just beyond the minimal focal distance of the IR lens, $0.5 \mathrm{~m}$, through an AR-coated ZnSe view-port. Data were collected for one experimental day using the full $320 \times 256$ frame, with a $2.5 \mathrm{~ms}$ integration time at $380 \mathrm{~Hz}$ and a second day using a $64 \times 256$ sub-window, with a 0.99 ms integration time at $1000 \mathrm{~Hz}$ (not discussed here). An example of the raw data is shown in Figure 1. The heated region of the foil is elongated vertically representing the change in emission along the poloidal field of view of the pinhole camera. The cutoff at the top of the image unfortunately prevents the diagnostic from imaging the lower divertor radiation. As discussed in Section IV, brightnesses measured on the poloidal cross-section are mapped to their tangency radii using the EFIT equilibrium and inverted assuming flux-surface symmetry to determine the 1D core emissivity profile. The signal increase shown in Figure 1b shows small scale variations not evident later in the shot in 1c. This is thought to be due to non-uniformity in the absorber construction which was blackened by hand using colloidal graphite spray. New IRVB absorber construction methods using physical vapor deposition result in more uniform coatings ${ }^{16}$.

\section{BENCH-TOP CALIBRATION AND TESTING}

Off-line calibrations are required to convert measured IR camera counts into temperature and confirm that the IRVB system is properly reporting power deposited on the absorber. While in-situ IRVB calibration systems have been demonstrated ${ }^{17}$, they are more cumbersome than the Ohmic replacement schemes used for $\mathrm{RBs}^{18}$. The C-Mod IRVB temperature calibration demonstrated a new, efficient method whereby a Solfadir non-uniformity correction (NUC) plate is located in place of the IRVB absorber and its temperature monitored by a thermocouple. Figure 2a shows the setup, where the NUC plate (right) is viewed by the IR camera (not shown) through the vacuum window (left) at the same focal position as the IRVB itself. The plate is heated and cooled and allowed to return slowly to room temperature over 10-15 minutes, collecting images intermittently over a temperature range of $10-35{ }^{\circ} \mathrm{C}$. Examples of the images collected are shown in Figure 2b,c for the $380 \mathrm{~Hz}, 2.5 \mathrm{~ms}$ integration time camera setup. For each pixel, an $n=2$ polynomial is fit to the curves of temperature versus camera counts derived from NUC data, as shown in Figure 3 for three pixel locations. A difference in these coefficients near pixel $(i, j)=(160,200)$ is observed, which is indicated by the yellow/orange region in the upper middle of Figure 2c. In this region, a failure and flaking of the anti-reflective coating was present, but out of focus. This highlights the importance for an image-wide calibration procedure, rather than performing an independent non-uniformity correction and then using a low-temperature blackbody at a single image location. By using the NUC as the blackbody source, the temperature and non-uniformity correction can be efficiently combined. This method assumes that the thermocouple accurately measures the NUC plate temperature. However, a small systematic $\Delta T$ offset has little consequence to the IRVB interpretation using (1). The time and space derivative terms are unaffected by this and the blackbody term shifts in magnitude by approximately $1 \% /{ }^{\circ} \mathrm{C}$ for typical ranges of IRVB temperature.

Also of note within the calibration data is the narrow range in temperature that can be measured before saturating the 14-bit camera, which occurs in two of the pixel locations in Figure 3. While IRVB and PFC surface thermography can utilize the same IR camera technology, their configurations are different. IRVBs measure small, few degree temperature changes and bin images in space and time to gain sufficient statistics. The integration time is set to the full intra-frame duration, here $2.5 \mathrm{~ms}$, while surface thermography configurations tend to have $1-10 \mu \mathrm{s}$ exposures and measure temperature increase of hundreds of degrees. This means that IRVBs are not benefiting from increases in camera frame rate and cannot benefit from increases in pixel count when they are realized by decreasing pixel pitch at fixed sensor size. These just distribute the same number of photons, $N$, between different frames and/or pixels, but when ultimately combined the signal to noise is still determined by $\sqrt{N}$ statistics.

Multiple sources have reported on the methods used to calibrate the $2 \mathrm{D}$ variation of the absorber properties ${ }^{1920}$. 


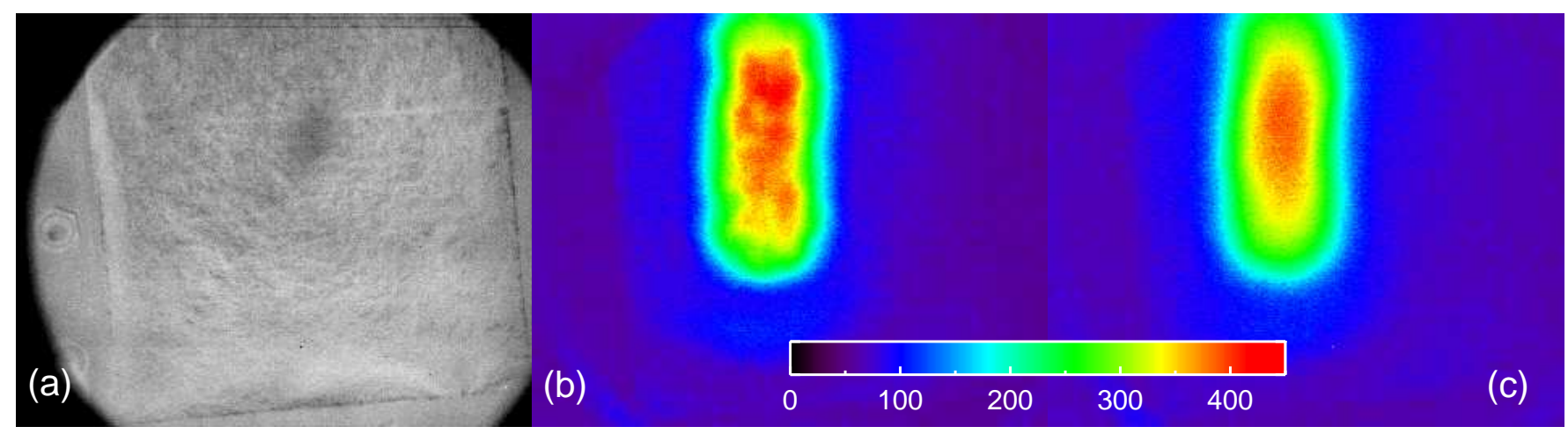

FIG. 1: Example of raw image data where (a) shows the absorber at $t=0$ while (b) and (c) show the in increase in IR camera counts relative to (a) at $t=1.05$, at peak temperature rise, and $t=1.5$, just prior to current ramp down.

A $405 \mathrm{~nm}$, BlueLyte laser of power $<5 \mathrm{~mW}$ was used to heat the absorber to confirm a previous calibration. This found that the average calibration values of $\kappa=10.9$ $\mathrm{mm}^{2} / \mathrm{s}, \varepsilon=0.85$ and $t_{f}=1.94 \mu \mathrm{m}$ best replicated the laser heating. The published value of $k=71.6 \mathrm{~W} / \mathrm{mK}$ was used. A scan in the laser power for $0.2 \mathrm{~Hz}$ square waves and a scan in the laser frequency, up to $64 \mathrm{~Hz}$, at high power can confirm both $t_{f}$ and $\kappa$. This is shown in Figure 4 using experimental data with a fixed laser power of $3.5 \mathrm{~mW}$. These two calibration terms are adjusted up and down by $20 \%$, resulting in either a frequency independent power mismatch or a frequency dependent power mismatch. There is good reason to believe that with a sufficiently dense spatial scan on the absorber the calibration uncertainty in (1) can effectively be reduced to the few percent level. It is thought that an additional scan in the focus of the laser heating would help to independently isolate the $\varepsilon$ value. Future work will demonstrate in more detail these suggested improvements to the IRVB absorber calibration approach necessary to support PEPC studies.

\section{C-MOD EXPERIMENTAL RESULTS}

This section outlines the analysis steps made to convert from measured temperature data to radiated power density profiles and total radiated power. Processing of the raw camera data indicated a $\sim 25 \mathrm{~Hz}$ oscillation that was persistent and nominally uniform across the camera with amplitude of $\sim 6$ counts. This was not due to the IRVB application and was isolated to be a camera issue of unknown origin. A digital band-stop filter and a 3-pt time averaging was used to remove this, but ultimately since the diagnostic relies on taking a time derivative, it impacts the quantitative noise-equivalent power density (NEPD) estimate. An additional slow rise of $\sim 60$ counts for $0<t<2.7$ seconds was observed, also not tied to the absorber and not correlated to any C-Mod magnet timing. This was removed by subtracting from the plasma viewing portion the signal derived from regions of the camera seeing the absorber, but where the absorber did not see plasma heating, such as the right side of images shown in Figure 1b,c, corresponding to the vertical slice of IRVB pixels at $i=250$ in Figure $5 \mathrm{a}$. These issues ultimately do not preclude the operation of the IRVB, but reflect practical environmental and camera noise issues encountered when operating IR systems near their limits. The IRVB showed no adverse response during 1.3 MA, 5.4 T full current disruptions where the chamber pressure rose to $>80$ mTorr, although it should be noted that the system was outside the TF magnets. The absorber was not electrically isolated from the machine ground, but no Ohmic heating in the foil was observed.

Once the temperature calibration has been applied, the IR camera data must be converted from $(i, j)$ camera pixels to $(m, n)$ bolometer pixels on which a finite-difference version of (1) can be computed. Unlike resistive bolometer systems where the concept of 'channels' is designed in, the IRVB can be rebinned based on the spatial structure or the signal to noise. Figure 5 a shows a rectangular, although non-uniform, grid as an example. The IRVB data are derived from the largest bolometer pixels centered at $i=145$. These are $30 \times 11$ camera pixels, matching the aspect ratio of the aperture. The absorber frame, evident in Figure 1a, is used to find the spatial conversion coefficient of 36.7 pixels $/ \mathrm{cm}$. The temperature and absorber calibration coefficients are averaged over these bolometer pixels and the power density of each component in (1) is computed. The time derivative uses a three-point Lagrangian interpolation, while the blackbody term can be computed directly from the data. The $x$ and $y$ components of the Laplacian are computed using a three-point central difference scheme that allows for non-uniform spacing ${ }^{21}$. Figure 5 b shows these time evolving components for one of the H-mode shots for a line of sight viewing through the core. It's clear the time-derivative is the strongest noise contributor, with an effective NEPD below $5 \mathrm{~W} / \mathrm{m}^{2}$. The raw data replicate features seen by other diagnostics, including the four distinct $\mathrm{H}$-modes from $0.68<t<1.12$ seconds and the laser ablation injection of tungsten at $t=1.37$. 

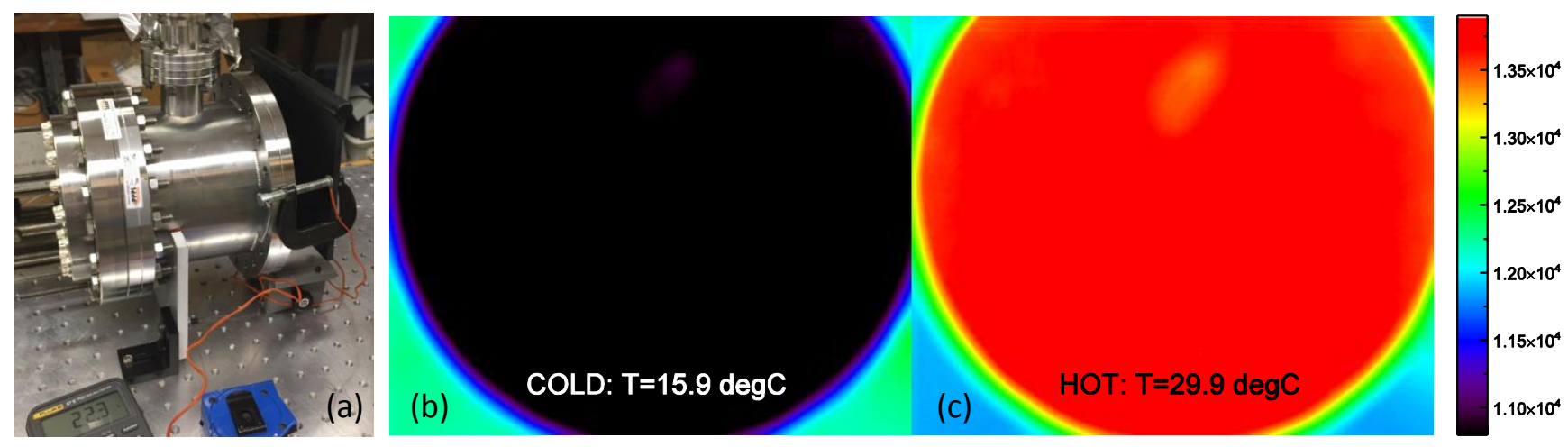

FIG. 2: Experimental setup of bench-top temperature calibration using a thermocouple-monitored non-uniformity correction plate (a). Example of full-frame temperature calibration data with the NUC plate cooled below (b) and heated above (c) room temperature.
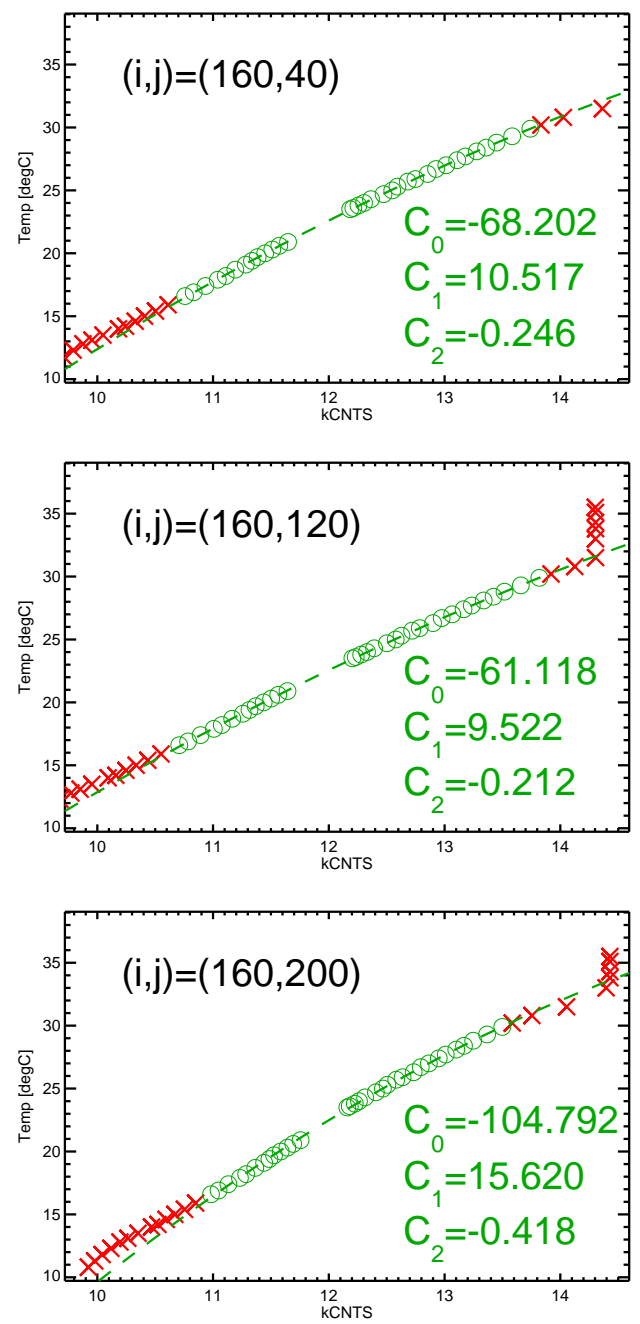

FIG. 3: Pixel-specific calibration data and resulting calibration polynomial curves for 2.5 ms integration time. Green circles are data used in fit, while red x's are additional data taken but excluded for being outside the range of operation of the IRVB. 


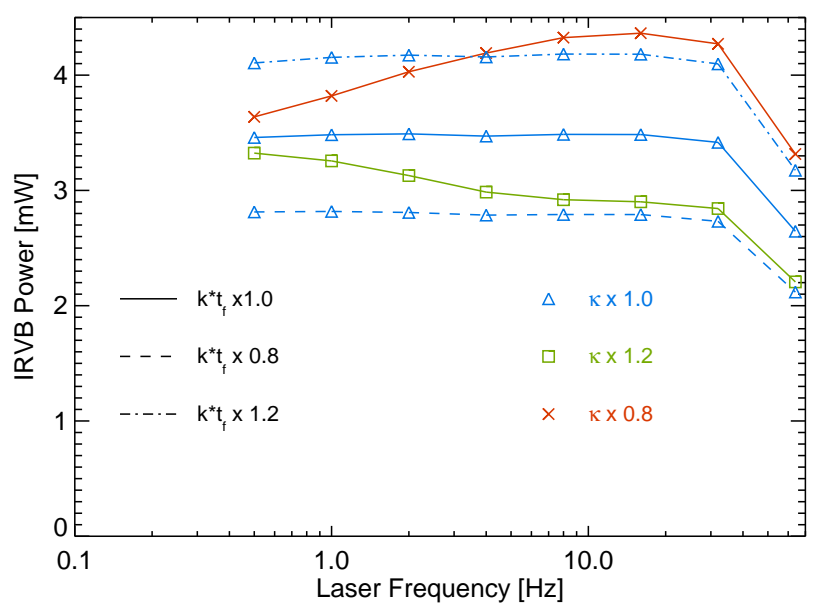

FIG. 4: Square wave laser heating of the absorber, showing how variation in the calibration constants changes the reconstructed laser power.
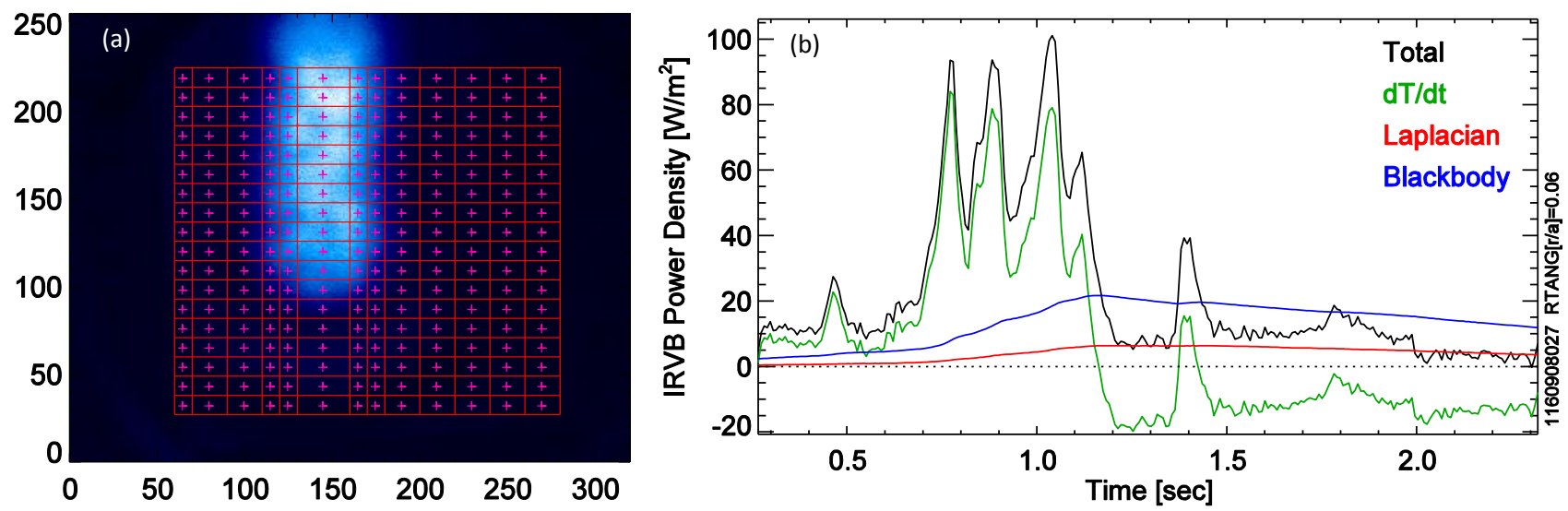

FIG. 5: Overlay of bolometer pixel grid on IRVB image (a) and comparison of power density terms derived from (1) for a core viewing IRVB channel

This analysis is replicated to form brightness profiles across the poloidal cross section, shown in Figure 6a. The IRVB system has a $\sim 4.5^{\circ}$ declination angle, but since an alignment calibration was not possible this angle was modified by less than $10 \%$ using an assumption the brightness is up/down symmetric in the core. These data are then inverted, assuming flux surface symmetry to derive the radiated power density, or emissivity, profiles, shown in Figure 6b. Times selected show profiles during various stages of the $\mathrm{H}$-mode as well as during the decay of the injected W. Profiles are hollow, consistent with historical observations during ELM-free $\mathrm{H}$-modes with total radiative power loss dominated by core intrinsic molybdenum emission.

Multiple metrics can be used to judge the accuracy of the IRVB radiated power results using comparison to C-Mod's existing radiated power diagnostic set ${ }^{22}$. A single channel RB of type ${ }^{23}$ and an AXUV-100G are given wide-angle views of the C-Mod plasma. Their signals are 'calibrated' by seeding discharges to radiative col- lapse and referencing those signals as equal to the input power. Details of core versus edge radiating structure can impact this relationship, but nominally these ' $2 \pi$ ' signals offer a crude, but useful control-room measure of C-Mod radiated power. The comparison shown in Figure $6 \mathrm{c}$ is encouraging, showing good agreement with the $2 \pi$ foil bolometer during the highly radiative $\mathrm{H}$-modes, and reasonable disagreement with the $2 \pi$ diode given the differences in spectral sensitivity between bolometers and AXUV diodes. In this plasma the total input power was 4.0 MW, with the $\mathrm{H} / \mathrm{L}$ back-transitions triggered as the plasma approaches $100 \%$ radiated power. Generally over the full run day, the IRVB levels remained consistent with both $2 \pi$ systems, although at times reported levels above or below both diagnostics. Resistive bolometers use $4 \mu \mathrm{m}$ $\mathrm{Au}$ absorbers while the IRVB uses a $2.5 \mu \mathrm{m}$ Pt foil, the reduction in thickness helping to increase the $\Delta T$. This thickness of $\mathrm{Au}$ has a $10 \%$ transmission at photon energies, $E_{\gamma}$ of approximately $7 \mathrm{keV}$, and at $6 \mathrm{keV}$ for the thinner Pt. Both will strongly absorb, the Mo radiation 

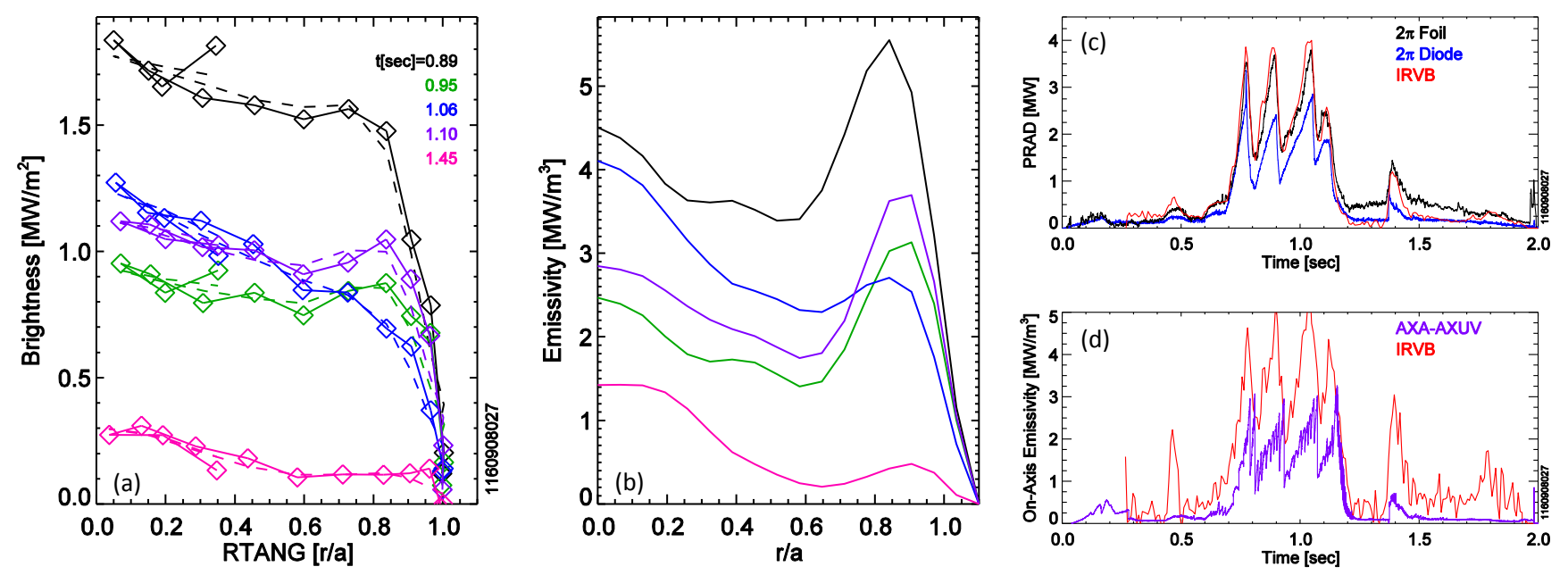

FIG. 6: Analyzed results showing brightness profiles (a), emissivity profiles (b) and comparison of IRVB to other measures of total radiated power (c) and on-axis emissivity (d)

from the plasma, which for peak $3<T_{e}<4 \mathrm{keV}$ results in dominant power loss for $E_{\gamma}<3 \mathrm{keV}$, with some higher-n emission in the $4<E_{\gamma}<5 \mathrm{keV}$ range.

Another comparison is made in Figure 6d, where the on-axis emissivity derived from IRVB is compared to that from AXUV diodes. In this case, it uses the AXA array, which views tangentially on the midplane allowing simple and reliable shell inversions. While the AXUV diodes have non-uniform spectral sensitivity preventing them from accurately reporting the total radiated power, the emission from the plasma core is generally close to that computed using $\mathrm{RBs}^{22}$. In this and all other cases, the IRVB claim on-axis emissivity values that are higher than AXUV diodes, sometimes as much as a factor of two, suggesting the IRVB emissivity profiles are more peaked.

Ideally, the radial emissivity profiles could be directly compared to the 16 -ch tangentially viewing $\mathrm{RB}$ diagnostic $^{22}$, but in these plasmas, there is significant electromagnetic interference from the ICRF heating system. Shown in Figure 7 are three RB chords from the low-field side (LFS) edge of the plasma, $R_{\text {edge }}=0.89 \mathrm{~m}$, to the core, $R_{o}=0.68 \mathrm{~m}$. During $0.6<t<1.1$ seconds, the system is dominated by noise from the $3-4 \mathrm{MW}$ of injected ICRF power. Outside of this time window, comparisons can be made, and Figure 7 overlays the time history of the brightness along these lines of sight computed from the IRVB-derived emissivity profiles. The coreviewing line of sight shows good agreement before and after the ICRF pulse when the diagnostic is reporting, while the LFS chords are under reported, especially after the H-mode. This under-reporting is consistent with the previous result that the IRVB profiles appear to be too peaked on-axis, but shows when averaging over the plasma the RB and IRVB are in reasonable agreement. Negative or near-zero brightness values, as shown for the $R_{T A N G}=0.80[\mathrm{~m}]$ chord in Figure7, are another indicator of a systematic error in the brightness profile. Fur- ther cross-validation requires more experimental time, in more different types of radiative scenarios then was possible with this brief test on C-Mod.

\section{SUMMARY}

These results show a successful test of an IRVB prototype to diagnose the temporal and spatial evolution of the radiation in the C-Mod tokamak plasma. Comparisons to existing radiation diagnostics such as AXUV diodes and resistive bolometers are favorable and the resilience against ICRF interference is clearly demonstrated. For this system, the accuracy of the IRVB to quantitatively reconstruct the radiated power is acceptable. But, improvement is still needed to support power exhaust research at the accuracy level, $<10 \%$, needed for activities like power balance where resistive bolometers remain the most commonly used tool.

\section{ACKNOWLEDGMENTS}

This work is supported in part by US Department of Energy award DE-AC05-00OR22725 and DESC0014264, using Alcator C-Mod, a DOE Office of Science User Facility. The authors would like to thank the diligent work of R. Rosati at MIT-PSFC to quickly install and remove the IRVB on Alcator C-Mod, enabling these results possible.

\section{REFERENCES}

\footnotetext{
${ }^{1}$ P.C. Stangeby A.W. Leonard. Nucl. Fusion., 51:063001, 2011.

${ }^{2}$ A. Loarte R. Neu. Fus. Eng. Design., 122:256, 2017.

${ }^{3}$ A.E. Jaervinen et al. J. Nucl. Mater., 463:135, 2015.
} 

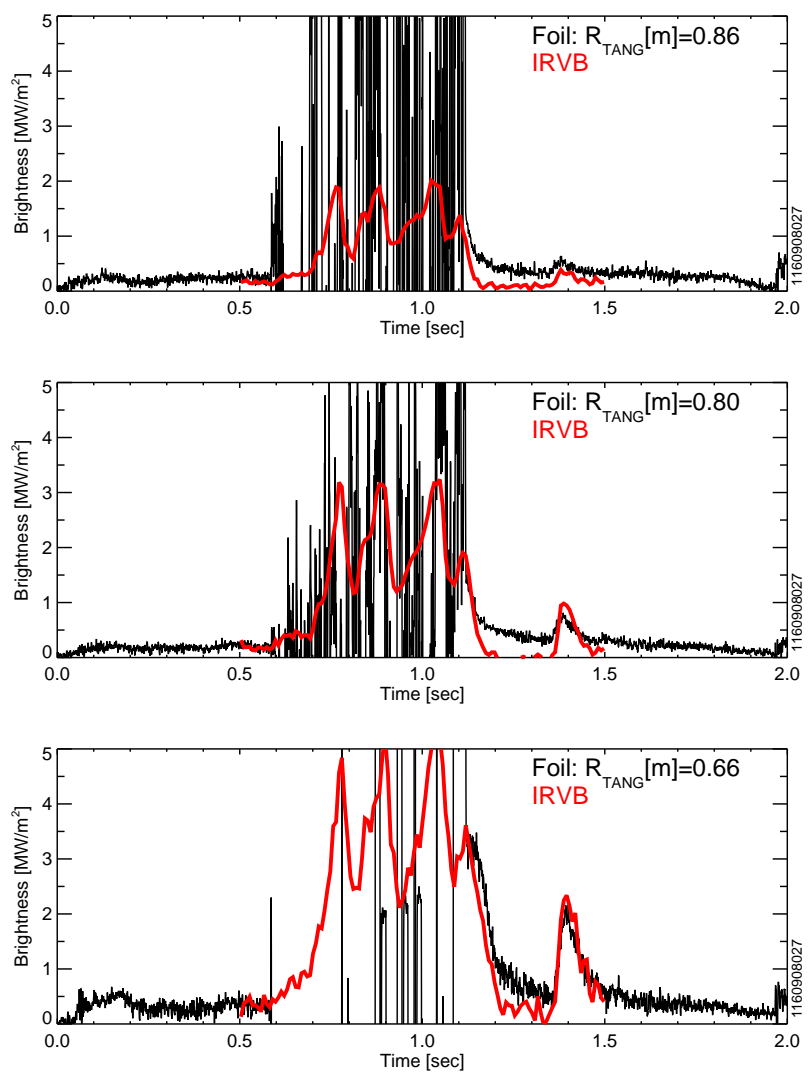

FIG. 7: Comparison of measured RB line-integrated brightness (black) with that derived from the IRVB emissivity profiles (red) for three viewing chords. ICRF induced noise from $0.6<t<1.1$ limits the RB system in this plasma

${ }^{4}$ F. Reimold et al. J. Nucl. Mater., 463:128, 2015.

${ }^{5}$ B.J. Peterson et al. J. Nucl. Mater., 363-365:412, 2007.

${ }^{6}$ J. Jang et al. Current Applied Physics., 18:461, 2018.

${ }^{7}$ J.M. Gao et al. Plasma Sci. Tech., 18:590, 2016.

${ }^{8}$ G.A. Wurden B.J. Peterson. Rev. Sci. Instrum., 70:255, 1999.

${ }^{9}$ B.J. Peterson et al. Rev. Sci.. Instrum., 72:923, 2001.

${ }^{10}$ R. Sano et al. Rev. Sci. Instrum., 87:053502, 2016.

${ }^{11}$ E. Marmer, et al. Fusion Sci. Tech., 51:261, 2007.

${ }^{12}$ K.F. Mast et al. Rev. Sci.. Instrum., 62:744, 1991.

${ }^{13}$ A.W. Leonard et al. Rev. Sci.. Instrum., 66:1201, 1995.

${ }^{14}$ B.J. Peterson et al. Rev. Sci.. Instrum., 74:2040, 2003.

${ }^{15}$ G.G. van Eden et al. Rev. Sci.. Instrum., 87:11D402, 2016.
${ }^{16}$ K. Mukai et al. Rev. Sci.. Instrum., 87:11E124, 2016.

${ }^{17}$ K. Mukai et al. submitted to Rev. Sci.. Instrum., 2018.

${ }^{18}$ A. Murari A. Hoffman. IEEE Trans. on Instrum. Measurement, 48:1183, 1999.

${ }^{19}$ S. Pandaya et al. Rev. Sci.. Instrum., 85:054902, 2014.

${ }^{20}$ R. Sano et al. Plasma Fusion Research, 7:2405039, 2012.

${ }^{21}$ J. Liu et al. Appl. Math Modelling, 19:162, 1995.

${ }^{22}$ M.L. Reinke I.H. Hutchinson. Rev. Sci.. Instrum., 79:10F306, 2008.

${ }^{23}$ E.R. Muller K.F. Mast. J. App. Phys., 55:2635, 1984. 